\title{
Redes semánticas poblacionales: un instrumento metodológico para la investigación educativa
}

\author{
Semantic networks of student populations: \\ a methodological tool for educational research
}

\author{
Sofía Judith Garófalo ${ }^{1}$ • Lydia Raquel Galagovsky² • \\ Manuel Alonso 3
}

\begin{abstract}
Resumen: Presentamos una propuesta metodológica cualitativa para indagar aprendizajes de una población de estudiantes. Utilizamos el concepto de red semántica para la representación gráfica de las respuestas individuales de los estudiantes frente a un problema determinado. Asimismo, el modelo de procesamiento de la información proporcionó la base teórico-práctica para la confección de los caminos cognitivos asociativos expresados por cada sujeto. Esta combinación teórico metodológica permitió elaborar redes semánticas poblacionales que representan la información acerca de los conocimientos declarativos de la población. Se propuso a estudiantes universitarios, de las asignaturas Biología y Química Biológica de distintas carreras, un problema de múltiples resoluciones acerca del metabolismo de los carbohidratos. Al ordenar las respuestas de todos los estudiantes en redes semánticas detectamos: (a) tendencias generales de errores arraigados que se repiten en cada asignatura, y (b) tendencias específicas de estrategias de respuestas o de errores que sólo aparecen en alguna de las poblaciones.

Palabras clave: Enseñanza superior. Metodología investigativa. Redes semánticas. Metabolismo de hidratos de carbono.
\end{abstract}

\begin{abstract}
We report a qualitative, innovative and original methodology for research in science education. We use the concept of Semantic Network as idea for displaying students' responses to a problem on a given topic. We proposed a problem with multiple resolutions on the metabolism of carbohydrates to university students of Biology and Biological Chemistry from different careers. Students' answers were organized in cognitive pathways, using a methodology derived from the Information Processing Model. Graphical representation of all individual cognitive pathways allowed us to construct semantic networks from each studied population. In this way the declarative knowledge of each population could be easily represented. These networks allowed us to detect: (a) answering strategies, (b) general trends of mistakes, and (c) mistakes that appeared in specific populations.
\end{abstract}

Keywords: Higher education. Research methodology. Semantic networks. Carbohydrate metabolism.

\footnotetext{
${ }^{1,3}$ Departamento de Ciencias Biológicas, Universidad de Buenos Aires (UBA), Ramos Mejía 841, C1405CAE, Ciudad de Buenos Aires, República Argentina. E-mail: <sjgarofalo@gmail.com>

${ }^{2}$ Instituto Centro de Formación e Investigación en Enseñanza de las Ciencias (CEFIEC), Facultad de Ciencias Exactas y Naturales, Universidad de Buenos Aires (UBA), Buenos Aires, República Argentina.
} 


\section{Introducción}

La investigación educativa constituye un campo complejo en el cual se integran y tensionan distintas disciplinas. En los últimos veinte años se ha desarrollado un considerable incremento de la cantidad y calidad de las investigaciones sobre fenómenos educativos (KEMMIS; MCTAGGART, 1988); sin embargo, es frecuente que los investigadores, inmersos en determinados paradigmas, fuercen instrumentos y técnicas metodológicas tradicionales, que no necesariamente son las más adecuadas para el objeto en estudio (TABER, 2007; TIKUNOFF; WARD; GRIFFIN, 1979).

Un problema constante asociado a la investigación sobre aprendizaje es la dificultad de acceder a los contenidos que están dentro de las inaccesibles estructuras cognitivas de los sujetos a partir de sus expresiones discursivas - sus conocimientos declarativos - (DE POSADA, 2002; MARÍN MARTÍNEZ, 2003; RYLE, 1949). Para reconocer categorías dentro de discursos o relatos, los investigadores aplican usualmente una lógica cualitativa de análisis conocida como "Grounded Theory" (GLASER, 1978), o Método Comparativo Constante (SIRVENT, 2006).

En este trabajo, se presenta una técnica innovadora para la representación de la información sobre los conocimientos declarativos de una población; es decir, sobre los discursos que han sido obtenidos empíricamente a partir de entrevistas. Se propone a las redes semánticas poblacionales como un nuevo instrumento para la investigación que, dentro de la metodología cualitativa, resuelve el problema de relevar la información proveniente de grupos de individuos, respetando simultáneamente las respuestas individuales.

\section{Las redes semánticas}

En los últimos años, un área de investigación en ciencias cognitivas se centró en generar modelos de procesamiento y evocación de la información de individuos. Para ello, generalmente se les solicitaba a los sujetos indagados que respondieran oralmente sobre asociaciones espontáneas que se les ocurrieran, por ejemplo, para armar categorías o relacionar información. Experimentos típicos requerían la evocación de palabras para encontrar eventuales organizaciones globales del lexicón (SIGMAN; CECCI, 2005). En ellos se detectaron formatos de respuestas que, organizados en grafos, mostraron estructuras de tipo "barrios" (small-world), con nexos entre ellos debidos a palabras polisémicas, tal como se muestra en la Figura 1. Un grafo es un dibujo constituido por nodos y relaciones entre nodos. Cada vértice es uno de los términos expresados y la relación semántica entre los nodos se representa mediante un trazo entre ellos, si es simétrica; y, si es asimétrica, se representa mediante una flecha (QUILLIAN, 1968; SHAPIRO; WODDMANSEE, 1969). El grafo completo se denomina red semántica.

Las redes semánticas surgieron como formatos para representar caminos de búsquedas cognitivas de información, mediante secuencias asociativas (WATTS; STROGATZ, 1998) que los científicos intentan modelizar. Los nodos de una red semántica no tienen sentido aislado, sino que muestran su significado cuando son vistos en relación a los otros conceptos con los cuales están conectados. Es decir, una red semántica es una representación gráfica de tipo proposicional del conocimiento de un sujeto.

Otras metodologías de búsquedas de tipo asociativo se aplican tanto para el armado y la localización de información en sistemas virtuales (tal como la "semantic Web”,www.w3.org), 
como para explicitar las interrelaciones entre hechos y condicionamientos almacenados en las memorias episódicas y semánticas de individuos (CALDERERO HERNÁNDEZ, 2003; TULVIN, 1972; VERA-NORIEGA; PIMENTEL; ALBUQUERQUE, 2005).

Figura 1. Formatos de grafos. (a) Grafos del tipo jerárquico; (b) grafos del tipo de pequeños barrios

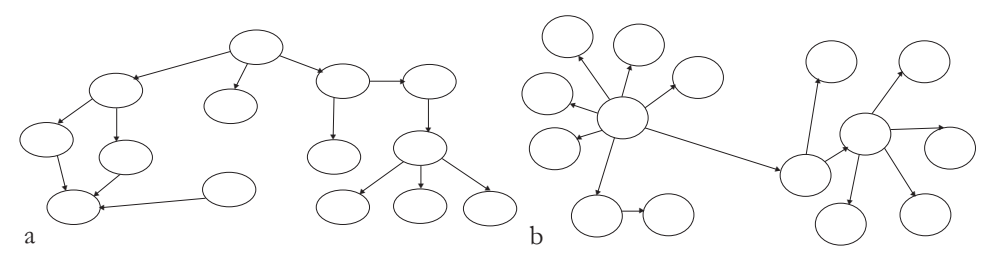

Fuente: esquema confeccionado por los autores.

Las redes semánticas se utilizan principalmente en el área de la lingüística para analizar cómo se logra la comunicación efectiva entre personas en el campo del conocimiento natural (SIGMAN; CECCI, 2005), o cómo se organiza el conocimiento de un sujeto que es experto en un área (MARCHIORI; LATORA, 2000). Dado que las redes semánticas son grafos, la información presentadas en ellas puede ser recuperada por programas de computación, o aplicárseles teorías matemáticas, para efectuar mediciones (COLLIPAL LARRÉ, 2002; HARJU, 2011; TAMAYO, 2009; VIVAS; COMESAÑA; VIVAS, 2007). Sin embargo, a pesar de los numerosos modelos matemáticos propuestos para interpretar la tridimensionalidad de las relaciones que se plasman en las redes semánticas obtenidas empíricamente, la aplicabilidad de los mismos no es general (STEYVERS; TENENBAUM, 2005).

\section{El problema central a investigar y la metodología empleada}

El presente trabajo es parte de una investigación amplia, en el marco de una Tesis Doctoral (GARÓFALO, 2010) centrada en comparar el acervo de conocimientos declarativos de cuatro poblaciones de estudiantes universitarios, sobre el metabolismo de hidratos de carbono. El tema es estructurante (GIORDAN et al., 1988) para la interpretación e integración de conocimientos bioquímicos, celulares y fisiológicos en los seres vivos; y numerosas investigaciones han demostrado que tanto estudiantes secundarios como universitarios tienen serias dificultades para su comprensión (BANET HERNÁNDEZ, 2001; FERREIRO; OCCELLI, 2008; LUZ, 2008; LUZ et al., 2008; OLIVEIRA et al., 2003; SONGER; MINTZES, 1994).

La investigación completa abarcó un total de 800 estudiantes de la Universidad de Buenos Aires, pertenecientes a las asignaturas Biología (del primer año de las carreras de Biología, Nutrición, Bioquímica, Medicina, Kinesiología y Psicología); Bioquímica Humana (del segundo año de la carrera de Nutrición de la Facultad de Medicina); Introducción a la Biología Celular y Molecular (del segundo año de la Licenciatura en Biología); y Química Biológica (del tercer año de las Licenciaturas en Biología o Química). En este trabajo se presentarán sólo los 
resultados obtenidos a partir de 182 estudiantes de la asignatura Biología y de 123 de Química Biológica. En todos los casos, la colaboración de los estudiantes con la investigación fue voluntaria y fuera del horario de clases.

La pregunta que movilizó la investigación estuvo basada en resultados preliminares que mostraban aprendizajes en los que faltaba la integración de conceptos (GARÓFALO, 2010), cuando se entrevistaba e indagaba la comprensión, en estudiantes universitarios que ya habían sido evaluados sobre el tema en cuestión (con exámenes parciales). Para estos estudios preliminares, se habían utilizado entrevistas abiertas, en las que cada sujeto debía resolver el problema presentado en el Cuadro 1 . Consideramos importante que la situación problemática seleccionada fuera sencilla, cotidiana, fácil de imaginar y que remitiera a la aplicación de conocimientos previos asociados a contenidos químicos, bioquímicos y/o fisiológicos. Debido a la extensión del tema, la indagación se centró en el metabolismo de los carbohidratos durante el período de absorción, en un organismo sano. Dada la posibilidad de obtener respuestas idiosincrásicas, se caracterizó al problema como de "múltiples resoluciones".

Cuadro 1. Problema de múltiples resoluciones

Una persona ingiere una porción de pizza. ¿Existe la posibilidad de encontrar alguno de los átomos de carbono que forma parte de la glucosa del almidón de esa pizza en la atmósfera? Menciona cómo y qué vías metabólicas estarían involucradas. Indica dónde esperarías encontrarlo.

Fuente: elaborado por los autores.

La indagación se había realizado con unos pocos individuos cuyas respuestas - grabadas y transcriptas - fueron posteriormente analizadas por metodología cualitativa con el Método Comparativo Constante (SIRVENT, 2006). Los resultados mostraron algunas regularidades provenientes de respuestas correctas, de errores y de respuestas incompletas. La investigación sobre una muestra considerablemente mayor de estudiantes condujo a un problema metodológico: cómo realizar el análisis cualitativo, con tal cantidad de respuestas diferentes. La aplicación de un software como el ATLAS-ti ${ }^{\circledR}$ no resultó adecuada porque permite localizar palabras y/o frases que deben determinarse de ante mano (BENCOMO; GODINO; WILHELMI, 2004; TAMAYO, 2009); por tanto, seleccionar este instrumento significaba perder la posibilidad de capturar la información referida a los diversos caminos resolutivos que surgían cuando los estudiantes se situaban frente al problema de múltiples resoluciones (Cuadro 1). Se decidió, pues, no automatizar una búsqueda por frases o conceptos, sino detectar posibles asociaciones, y el orden jerárquico de los conceptos empleados por cada individuo al responder al problema de múltiples resoluciones.

La búsqueda bibliográfica para hallar una solución a esta dificultad metodológica condujo a utilizar el concepto de red semántica como idea de representación gráfica de la respuesta de cada individuo (MÉRIDA, 2004; WATTS; STROGATZ, 1998), y el modelo de procesamiento de la información (ERICSSON; SIMON, 1999) como base teórico-práctica para la confección de los caminos cognitivos asociativos expresados por cada sujeto. Esta combinación teórico 
metodológica permitió superar la mencionada dificultad al crear lo que denominamos redes semánticas poblacionales. A continuación se presentarán los fundamentos teóricos del modelo de procesamiento de la información y la metodología práctica para organizar los caminos cognitivos individuales y, a partir de éstos, las redes semánticas poblacionales.

\section{Técnica de entrevistas concurrentes desde el modelo de procesamiento de la información}

Este modelo supone que para que una determinada información llegue a formar parte del conocimiento de una persona en la memoria a largo plazo debe atravesar el filtro sensorial y la memoria a corto plazo. La memoria de trabajo (JOHNSTONE, 1997) efectúa un monitoreo entre la nueva información y la ya existente en la memoria de largo plazo. Por consiguiente, cualquier información externa que se sume a la memoria a largo plazo está condicionada por el filtro sensorial, por la capacidad retentiva de la memoria de corto plazo y por el conocimiento previo que tenga el sujeto.

Según el modelo de Ericsson y Simon (1999), la información no es guardada en forma arbitraria en la memoria a largo plazo, sino que se jerarquizaría en "señaladores" al vincular contenidos nuevos con información previa, formando redes cognitivas de asociación. Cuando una persona se enfrenta a una pregunta, la necesidad de generar una respuesta lo lleva a evocar información guardada en la memoria a largo plazo. La información sería accesible debido a que el sujeto habría reconocido en la pregunta algún estímulo que utiliza como "anzuelo" en su búsqueda cognitiva para asociar con algún "señalador". Esto implicaría que en los procesos de búsqueda y evocación de una respuesta, se establecen construcciones asociativas "anzuelo-señalador". Tales construcciones son reflejo del material informativo recuperado desde la memoria a largo plazo, organizado semánticamente en función de las habilidades cognitivas idiosincrásicas de cada sujeto.

Ericsson y Simon (1999) propusieron, además, dos tipos de entrevistas: las entrevistas concurrentes, para detectar cadenas asociativas "anzuelo-señalador"; y las entrevistas recurrentes, que permiten indagar acerca de los significados otorgados por los sujetos. Frente a las entrevistas concurrentes, cada sujeto debe verbalizar todos los pensamientos que aparecen en su atención como parte de la resolución de la tarea que se le plantea. En esta técnica, denominada "pensar en voz alta", el entrevistador no debe intervenir haciendo comentarios ni nuevas preguntas, durante el tiempo que involucre la resolución del problema. Este es el tipo de entrevistas que se aplicaron para la obtención de los datos del presente trabajo.

\section{Respuestas al problema de múltiples resoluciones}

El problema planteado a los estudiantes (Cuadro 1) tenía como característica principal la posibilidad de ser respondido por "sî" o por "no", con explicaciones diferentes e idiosincrásicas. En la Tabla 1, se encuentran los porcentajes de cada respuesta dada por los estudiantes de las asignaturas Biología y Química Biológica respectivamente. Aquellos estudiantes que respondieron afirmativamente al problema, espontáneamente esgrimieron argumentos como justificación; en cambio, los que dieron respuestas negativas no efectuaron argumentación alguna. Estos datos revelaron graves fallas en el aprendizaje de la temática, particularmente en la materia Biología. 
Garófalo, S. J.; Galagovsky, L. R.; Alonso, M.

Tabla 1. Porcentaje de estudiantes de Biología y de Química Biológica que negaron o que afirmaron la posibilidad planteada en el problema de múltiples resoluciones*

$\%$ de estudiantes que negaron $\%$ de estudiantes que afirmaron la

la posibilidad planteada en el posibilidad planteada en el problema de

problema de múltiples resoluciones múltiples resoluciones y dieron argumentos

182 estudiantes

de Biología

123 estudiantes

de Química

Biológica
$66 \%$

(121 estudiantes)

$10 \%$

(12 estudiantes)
$34 \%$

(61 estudiantes) $90 \%$

(111 estudiantes)

${ }^{*}$ El problema fue respondido por 305 estudiantes en total.

Fuente: elaborado por los autores.

Realizar un análisis del contenido de las respuestas significaba sistematizar la información poblacional proveniente de los 61 estudiantes de Biología y de los 111 de Química Biológica, sin perder la información cualitativa de los reportes individuales. Un primer intento fue analizar las respuestas organizando categorías, a partir del método comparativo constante (SIRVENT, 2006), pero la operación no resultó apropiada por dos causas interrelacionadas. Por un lado, el método comparativo constante supone un universo infinito de posibles respuestas sobre las que se analizan emergentes como regularidades encontradas (categorías). En cambio, si bien las entrevistas concurrentes permiten la libre organización discursiva de los encuestados, y el problema de múltiples resoluciones es un problema abierto, el conjunto de posibles respuestas correctas no es infinito. Por otra parte, en el método comparativo constante no deberían establecerse juicios de valor sobre la exactitud o error de los datos empíricos obtenidos - que generalmente son opiniones de los encuestados. Sin embargo, al aplicarlo a las respuestas de las entrevistas concurrentes sólo se encontraron categorías emergentes tales como respuestas correctas, incorrectas o incompletas. En estos casos, además, se perdía la información cualitativa sobre respuestas individuales. Debido a estas consideraciones previas, se planteó utilizar otros marcos metodológicos que se describen a continuación.

\section{Construcción del camino resolutivo de cada individuo y de las redes semánticas poblacionales}

El problema de múltiples resoluciones inducía a una respuesta con ilación conceptual de tipo secuencial, considerando las oraciones como unidades lingüísticas con independencia sintáctica y semántica (HART JR.; KRAUT, 2007; KINTSCH, 1974, 1988). A su vez, de acuerdo al marco teórico del modelo de procesamiento de la información (ERICSSON, 2006; ERICSSON; SIMON, 1999), cada individuo organizaría su respuesta como relaciones asociativas anzuelo-señalador. A partir de estas consideraciones, rescatamos las respuestas expresadas oralmente para construir el camino resolutivo que daba cuenta del conocimiento declarativo de cada sujeto entrevistado. Así, la respuesta de cada estudiante fue plasmada gráficamente. El 
camino resolutivo representa la secuencia lineal-temporal de información expresada por el sujeto (su conocimiento declarativo). A modo de ejemplo, se mencionan algunas respuestas de los estudiantes frente al problema de múltiples resoluciones, y se detallan los caminos resolutivos confeccionados a partir de los conceptos centrales de las unidades proposicionales rescatadas.

a) Ejemplo de un estudiante de la asignatura Biología (respuesta del estudiante $\mathrm{N}^{\mathrm{o}} 72$ ):

La pizza contiene almidón y durante el proceso digestivo es eliminado metano por la actividad bacteriana. De ahí el $\mathrm{CO}_{2}$ que sale a la atmósfera... Es la única respuesta que se me ocurre.

Camino resolutivo: pizza $\rightarrow$ almidón $\rightarrow$ proceso digestivo $\rightarrow$ metano $\rightarrow$ eliminación de $\mathrm{CO}_{2}$ a la atmósfera diante $\left.\mathrm{N}^{\mathrm{o}} 2\right)$ :

b) Ejemplo de un estudiante de la asignatura Química Biológica (respuesta del estuEl carbono viene del $\mathrm{CO}_{2}$ que proviene de la respiración celular y se elimina a la atmósfera... Pero la verdad, jno tengo idea cómo sale! Camino resolutivo: $\mathrm{C}-\mathrm{CO}_{2} \rightarrow$ respiración celular $\rightarrow$ eliminación de $\mathrm{CO}_{2}$ a la atmósfera $\rightarrow$ no sabe cómo sale

$\mathrm{Al}$ analizar los caminos resolutivos individuales en cada población, se encontraron regularidades que permitieron organizar gráficamente la totalidad de estos caminos en un nuevo instrumento que denominamos red semántica poblacional. Esta red expresa la secuencia y la frecuencia con que cada nodo apareció en cada camino individual, observándose pocos nodos iniciales de mayor frecuencia de aparición y una mayor variabilidad en los nodos subsiguientes. Las redes poblacionales se confeccionaron con las respuestas de los estudiantes que contestaron afirmativamente al problema de múltiples resoluciones y dieron argumentos (Tabla 1). La red poblacional correspondiente a los estudiantes de Biología se muestra en la Figura 2, y la de los estudiantes de Química Biológica se presenta distribuida en las Figuras 3a y 3b.

En cada red (Figuras 2, 3a y 3b), con un número, se identifica la frecuencia de uso de cada nodo; los números de los nodos del extremo derecho de cada camino indican la frecuencia de uso de ese camino. Por ejemplo, el primer camino en la Figura 2 fue utilizado por 19 estudiantes de un total de 61 que respondieron afirmativamente el problema de múltiples resoluciones y argumentaron su respuesta. Asimismo, la diversidad de respuestas pudo ser incluida como ramificaciones de los caminos. Por ejemplo, en la Figura 3a, si bien 47 estudiantes comenzaron con el nodo $\mathrm{C}-\mathrm{CO}_{2}$, sucesivas bifurcaciones condujeron a siete caminos resolutivos finales diferentes.

$\mathrm{Al}$ ordenar en redes semánticas poblacionales las respuestas de todos los estudiantes, se obtuvo una "foto" de la información que esa muestra poblacional expresaba sobre el tema indagado (en este caso, el metabolismo de los hidratos de carbono), en ese momento específico. Es decir, se representaron las evidencias empíricas sobre el acervo de conocimientos de cada población. 
Garófalo, S. J.; Galagovsky, L. R.; Alonso, M.

Figura 2. Red semántica resultante del análisis de las entrevistas concurrentes de los estudiantes de Biología

\begin{tabular}{|c|c|c|c|c|c|}
\hline $\begin{array}{l}\mathrm{C}-\mathrm{CO}_{2} \\
(19)\end{array}$ & $\begin{array}{l}\text { Producto d } \\
\text { de la respir }\end{array}$ & $\begin{array}{l}\text { el Ciclo de Krebs } \\
\text { ción celular (19) }\end{array}$ & $\rightarrow$ & $\begin{array}{l}\text { Eliminación de } \mathrm{CO}_{2} \\
\text { a la atmósfera (19) }\end{array}$ & \\
\hline $\begin{array}{l}\text { Almidón } \rightarrow \\
\text { (11) }\end{array}$ & $\begin{array}{l}\text { Glucosa } \rightarrow \\
\text { (11) }\end{array}$ & $\begin{array}{l}\text { Distribución } \\
\text { según necesidades } \\
\text { (11) }\end{array}$ & $\rightarrow \underset{\mathrm{Ca}}{\mathrm{Ca}}$ & $\begin{array}{l}\text { ólisis/Ciclo de Krebs/ } \rightarrow \\
\text { na respiratoria }\end{array}$ & $\begin{array}{l}\text { Eliminación de } \\
\mathrm{CO}_{2} \text { a la atmósfera } \\
(11)^{2}\end{array}$ \\
\hline
\end{tabular}

Pizza $\rightarrow \begin{aligned} & \text { Almidón } \\ & (31)\end{aligned} \rightarrow \begin{aligned} & \text { Proceso } \\ & \text { digestivo } \\ & (31)\end{aligned}$
$\begin{aligned} & \text { Megún necesidades } \\ & (20)\end{aligned}$
$\begin{aligned} & \text { Metano } \\ & (11)\end{aligned}$ $\begin{aligned} & \begin{array}{l}\text { Glucólisis/Ciclo } \\ \text { de Krebs/Cadena } \\ \text { respiratoria } \\ (20)\end{array} \\ & \begin{array}{l}\text { Eliminación de } \\ \mathrm{CO}_{2} \text { a la atmósfera } \\ (20)^{2}\end{array}\end{aligned}$

Los números entre paréntesis representan las frecuencias con que apareció el nodo. Fuente: elaborado por los autores

Figura 3a. Parte de la red semántica resultante del análisis de las Entrevistas Concurrentes de los estudiantes de Química Biológica. Se presentan los caminos cognitivos que partieron de los nodos iniciales "C-CO2" y "Persona"

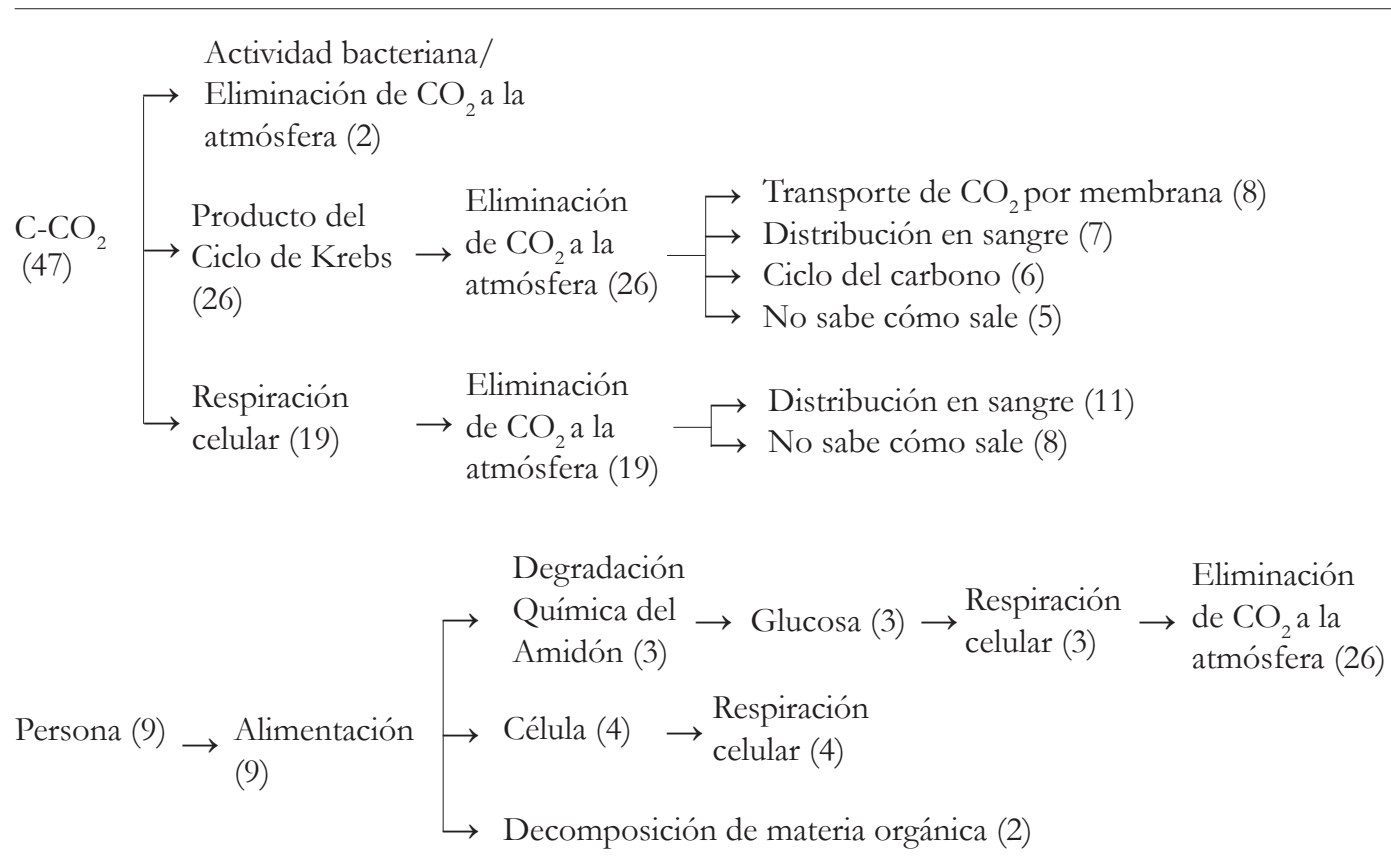

Los números en cada nodo, entre paréntesis, representan las frecuencias con que apareció.

Fuente: elaborado por los autores. 
Figura 3b. Parte de la red semántica resultante del análisis de las Entrevistas Concurrentes de los estudiantes de Química Biológica. Se presentan los caminos cognitivos que partieron del nodo inicial "Pizza"

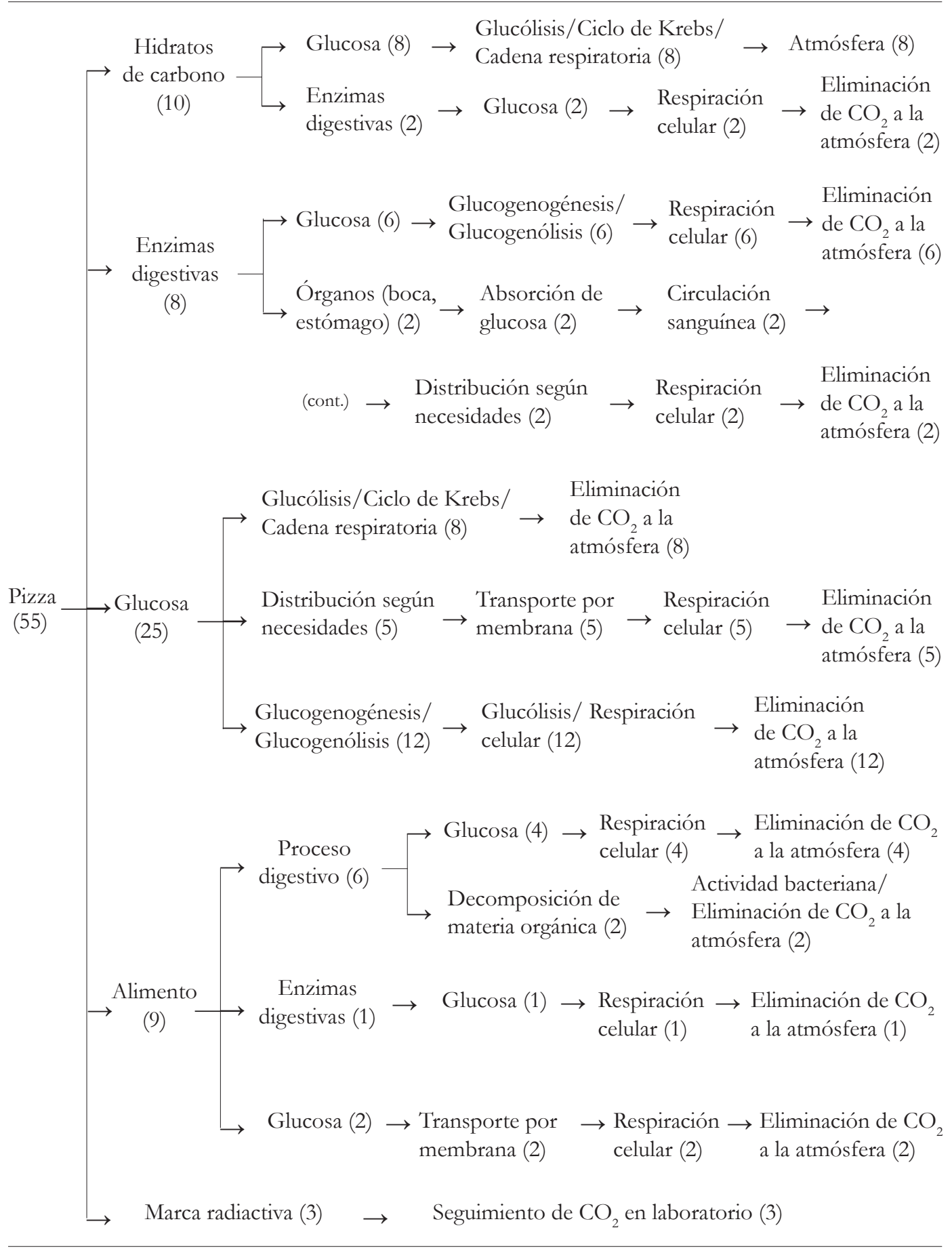

Los números en cada nodo, entre paréntesis, representan las frecuencias con que apareció.

Fuente: elaborado por los autores. 


\section{Análisis del conocimiento declarativo a partir de las Redes Semánticas poblacionales}

La comparación cualitativa entre las Figuras 2, 3a y 3b revela claramente la diferencia de complejidad en el contenido de las respuestas entre las poblaciones de estudiantes de la asignatura Biología (primer año de la universidad) y de la asignatura Química Biológica (tercer o cuarto año de las carreras de Biología o Química). Esta variación tiene un correlato directo con el tiempo asignado a las clases de metabolismo de hidratos de carbono dentro cada asignatura y con la bibliografía respectiva. Por un lado, en la materia Biología el tema de metabolismo de carbohidratos se dictó en dos clases de tres horas cada una, y la bibliografía de consulta fue el texto de Curtis y Sue Barnes (2000). Por otro lado, el dictado en la asignatura Química Biológica demandó 6 clases teóricas de tres horas cada una, y se utilizó como bibliografía de consulta principal el libro de Stryer, Berg y Tymoczko (2003).

Tanto en la asignatura Biología como en Química Biológica se enseñan las vías metabólicas de glucólisis, respiración celular aeróbica y fermentación, pero con distintos grados de profundidad. En Química Biológica, también se enseñan gluconeogénesis, glucogenogénesis, glucogenolisis, adaptaciones del metabolismo de los carbohidratos a los distintos estados nutricionales de un organismo, la regulación de la glucemia, los efectos de la insulina y glucagón, y una clase de integración metabólica. Estas diferencias en cuanto al contenido enseñado se ven reflejadas en las respectivas redes semánticas poblacionales, donde la de Química Biológica se muestra mucho más rica en detalles de procesos que la de Biología (comparar Figuras, 3a y 3b).

El valor de la red semántica poblacional, además de poner en evidencia el acervo de conocimientos de cada población acerca del metabolismo en cuestión, reside en que reveló similitudes y diferencias. Llama la atención, por ejemplo, tendencias erróneas sobre caminos resolutivos en ambas poblaciones:

a) 11 estudiantes de Biología asociaron el proceso digestivo con la producción de metano (camino resolutivo 4, Figura 2) y la subsiguiente eliminación de $\mathrm{CO}_{2}$ a la atmósfera, poniendo en evidencia una inconsistencia conceptual.

b) 2 estudiantes de Química Biológica asociaron la producción de $\mathrm{CO}_{2}$ con la actividad bacteriana (camino resolutivo 1, Figura 3a y otros 2 relacionaron el proceso digestivo con la actividad bacteriana para la producción de $\mathrm{CO}_{2}$ (camino resolutivo 9, Figura 3b).

c) 4 estudiantes de Química Biológica utilizaron el término impreciso “descomposición de la materia orgánica" (camino resolutivo 10, Figura 3a y camino resolutivo 9, Figura 3b).

d) 31 estudiantes de Biología utilizaron la expresión "la glucosa se distribuye hacia donde más se necesita" (caminos resolutivos 2 y 3, Figura 2); y, en forma similar, respondieron 7 estudiantes de Química Biológica (caminos resolutivos 4 y 6, Figura 3b). Esto significa que, un 51\% de los estudiantes de Biología y un 6\% de los estudiantes de Química Biológica pusieron en evidencia supuestos de intencionalidad sobre la captación-distribución de glucosa para los diferentes tejidos.

Por otro lado, hubo expresiones específicas en Química Biológica, relacionadas con mayor cantidad de información respecto de lo que se enseña en Biología:

a) 18 estudiantes expresaron una necesaria secuencia previa de los procesos de glucogenogénesis y glucogenolisis antes de la respiración celular (caminos resolutivos 3 y 7, Figura 
3b). Esto significa que el 16\% de los estudiantes de Química Biológica no consideró que estas vías son alternativas y que dependen del estado nutricional del organismo.

b) 13 estudiantes de los que comenzaron con el nodo $\mathrm{C}-\mathrm{CO}_{2}$, terminaron su argumentación manifestando una reflexión autoevaluativa: plantearon desconocer cómo se elimina el $\mathrm{CO}_{2}$ producido por la respiración celular (8 estudiantes) o por el ciclo de Krebs (5 estudiantes) (caminos resolutivos 5 y 7 , Figura $3 \mathrm{a}$ ).

c) 3 estudiantes mencionaron la utilización de marca radiactiva como un posible método para resolver el problema, hecho relacionado con la interpretación de protocolos de investigación como parte de los contenidos estudiados en la materia (camino resolutivo 12, Figura 3b).

\section{Consideraciones finales}

El presente trabajo es una propuesta metodológica innovadora y original para enfrentar el desafío de indagar conocimientos acerca de un tema, en una población particular, partiendo de las respuestas individuales expresadas en entrevistas. Tal desafío se abordó mediante la generación de un problema de múltiples resoluciones, su aplicación en entrevistas concurrentes en el marco metodológico del modelo de procesamiento de la información, y la representación de las respuestas como caminos resolutivos, dentro del marco teórico de tal modelo.

La utilización de un mismo instrumento - como el problema de múltiples resoluciones - y de una misma técnica de entrevistas - como las entrevistas concurrentes - tuvo como fundamento poder contar con la posibilidad de comparar los conocimientos declarativos de los sujetos sobre un dado tema, más allá de la amplitud o profundidad con que hubiera sido enseñado. Esto señala la potencialidad de la propuesta para realizar análisis comparativos entre poblaciones, acerca de sus conocimientos sobre un mismo tema. Este procedimiento puede ser aplicado a numerosos contenidos cuando se logre diseñar un problema de múltiples resoluciones que pueda ser respondido por sujetos con diferentes niveles de instrucción.

Frecuentemente, los estudiantes no integran sus conocimientos parciales en marcos contextuales, pues tanto la enseñanza como en la evaluación se presentan preguntas específicas, o ejercicios que ellos resuelven más por entrenamiento que por comprensión conceptual integradora. Es importante, por tanto, que el problema de múltiples resoluciones sea un disparador potente de las entrevistas concurrentes y permita que los estudiantes aborden su resolución desde ángulos inéditos que permeen sus estructuras cognitivas "entrenadas en responder adecuadamente", y liberen razonamientos espontáneos. Un buen diseño del problema de múltiples resoluciones es crítico para hacer emerger evidencias de aprendizaje, que no suelen aparecer en las evaluaciones tradicionales. La idea fundamental es que el problema requiera ser respondido desde una visión integradora de algún tópico, demandando a los estudiantes insertar sus aprendizajes generalmente fragmentados en contextos articulados. La reconstrucción de los caminos cognitivos idiosincrásicos utilizados por cada estudiante para resolver un problema de múltiples resoluciones es una técnica sencilla. El mayor requerimiento resulta de haber aplicado correctamente la técnica de entrevistas recurrentes. 
Garófalo, S. J.; Galagovsky, L. R.; Alonso, M.

Contar con las redes semánticas poblacionales, como una imagen representativa del acervo de conocimientos declarativos de cada población, sobre un tema específico, permite dar cuenta de:

a) Tendencias generales de errores arraigados y persistentes que se repiten en cada población, a pesar de la diferente profundidad en su respectiva enseñanza;

b) Tendencias específicas de estrategias de respuestas o de errores que sólo aparecen en alguna de las poblaciones.

De esta forma, la presente propuesta constituye un aporte innovador para la obtención de información empírica relevante sobre los conocimientos declarativos y su necesaria correlación con los aprendizajes efectivos. Puede utilizarse para hacer evidentes las asociaciones cognitivas heurísticas e idiosincrásicas latentes en cada población de estudiantes, y realizar estudios comparativos entre poblaciones.

Finalmente, todas las consideraciones específicas acerca del aprendizaje del metabolismo de los hidratos de carbono, presentadas en este trabajo, abren puertas para la revisión crítica sobre la enseñanza de este tema (GARÓFALO; ALONSO; GALAGOVSKY, 2014; GARÓFALO; GALAGOVSKY; ALONSO, 2014).

\section{Agradecimientos}

Este trabajo fue financiado con los subsidios de la Universidad de Buenos Aires UBACyT X181 y UBACyT U011. S. J. G. fue becaria de doctorado de la Universidad de Buenos Aires (2004-2009).

\section{Referencias}

BANET HERNÁNDEZ, E. Los procesos de la nutrición humana. Madrid: Síntesis, 2001.

BENCOMO, D.; GODINO, J. D.; WILHELMI, M. R. Elaboración de redes ontosemióticas de configuraciones didácticas con ATLAS/TI. In: CAÑAS, A. J.; NOVAK, J. D.; GONZÁLEZ, F. M. (Ed.). Concept maps: theory, methodology, technology: proceedings of the first International Conference on Concept Mapping. Pamplona: Universidad Pública de Navarra, 2004. Disponible en: <http://cmc.ihmc.us/papers/cmc2004-135.pdf >. Acceso el: 29 enero 2015.

CALDERERO HERNÁNDEZ, J. Estudio de libros de texto de ciencias de la naturaleza mediante análisis cuantitativo basado en la teoría de grafos. 2003. 320 h. Tesis Doctoral - Departamento de Didáctica de las Ciencias Experimentales, Facultad de Educación, Universidad Complutense de Madrid, Madrid, 2003. Disponible en: <http:// biblioteca.ucm.es/tesis/edu/ucm-t26700.pdf>. Acceso el: 29 enero 2015. 
COLLIPAL LARRÉ, E. Conceptualización a través de redes semánticas naturales de los módulos de autoaprendizaje en anatomía humana. Revista Chilena de Anatomía, Temuco, v. 20, n. 1, p. 1-8, 2002. Disponible en: < http://dx.doi.org/10.4067/S071698682002000100009>. Acceso el: 29 enero 2015.

CURTIS, H.; SUE BARNES, N. Biología. 6. ed. Buenos Aires: Médica Panamericana, 2000.

DE POSADA, J. M. Memoria, cambio conceptual y aprendizaje de las ciencias. Revista Electrónica de Enseñanza de las Ciencias, Vigo, v. 1, n. 2, p. 92-113, 2002. Disponible en: <http://reec.uvigo.es/volumenes/volumen1/REEC_1_2_4.pdf>. Acceso el: 29 enero 2015.

ERICSSON, K. A. Protocol analysis and expert thought: concurrent verbalizations of thinking during experts' performance on representative task. In: ERICSSON, K. A. et al. (Ed.). Cambridge handbook of expertise and expert performance. Cambridge: Cambridge University Press, 2006. p. 223-242.

ERICSSON, K. A.; SIMON, H. A. Protocol analysis: verbal reports as data. Cambridge: MIT Press, 1999.

FERREIRO G.; OCCELLI, M. Análisis del abordaje de la respiración celular en textos escolares para el ciclo básico unificado. Revista Electrónica de Enseñanza de las Ciencias, Vigo, v. 7, n. 2, p. 387-398, 2008. Disponible en: <http://reec.uvigo.es/ volumenes/volumen7/ART7_Vol7_N2.pdf>. Acceso el: 29 enero 2015.

GARÓFALO, S. J. Análisis de obstáculos en el aprendizaje de metabolismo de hidratos de carbono: un estudio transversal. 2010. 506 h. Tesis doctoral (Química Biológica) - Facultad de Ciencias Exactas y Naturales, Universidad de Buenos Aires, Buenos Aires, 2010. Disponible en: <http://digital.bl.fcen.uba.ar/Download/Tesis/Tesis_4772_Garofalo. pdf>. Acceso el: 29 enero 2015.

GARÓFALO, S. J.; ALONSO, M.; GALAGOVSKY, L. R. Nueva propuesta teórica sobre obstáculos epistemológicos de aprendizaje: el caso del metabolismo de los carbohidratos. Enseñanza de las Ciencias, Barcelona, v. 32, n. 3, p. 155-171, 2014. Disponible en: <http://ddd.uab.cat/pub/edlc/edlc_a2014v32n3/edlc_a2014v32n3p155.pdf>. Acceso el: 29 enero 2015.

GARÓFALO, S. J.; GALAGOVSKY, L. R.; ALONSO, M. Dificultades en el aprendizaje del metabolismo de los carbohidratos: un estudio transversal. Química Viva, Buenos Aires, v. 13, n. 1, p. 31-55, 2014. Disponible en: <http://www.quimicaviva.qb.fcen.uba.ar/v13n1/ garofalo.pdf $>$. Acceso el: 29 enero 2015.

GIORDAN, A. et al. Conceptos de biología 1. La respiración. Los microbios. El ecosistema. La neurona. Barcelona: Labor, 1998.

GLASER, B. G. Theoretical sensitivity: advances in the methodology of grounded theory. Mill Valley: The Sociology Press, 1978.

HARJU, T. Lecture notes on graph theory. Turku: University of Turku, Finlandia, 2011. Disponible en: <http://users.utu.fi/harju/graphtheory/graphtheory.pdf>. Acceso el: 29 enero 2015. 
Garófalo, S. J.; Galagovsky, L. R.; Alonso, M.

HART JR., J.; KRAUT, M. A. Neural hybrid model of semantic object memory (version 1.1). In: (Ed.). Neural basis of semantic memory. Cambridge: Cambridge

University Press, 2007. p. 331-360. Disponible en: < http://dx.doi.org/10.1017/

CBO9780511544965.014>. Acceso el: 29 enero 2015.

JOHNSTONE, A. H. J. Chemical teaching: science or alchemy? Journal of Chemical Education, Easton, v. 74, n. 3, p. 262-268, 1997. Disponible en: <http://gesn.ch/mat_ didattico/teoria/Johnstone.pdf>. Acceso el: 29 enero 2015.

KEMMIS, S.; MCTAGGART, R. Cómo planificar la investigación acción. Barcelona: Laertes, 1988.

KINTSCH, W. The representation of meaning in memory. Hillsdale: Lawrence Erlbaum, 1974.

. The role of knowledge in discourse comprehension: a construction-integration model. Psychological Review, Washington, v. 95, n. 2, p. 163-182, 1988.

LUZ, M. R. Glucose as the sole metabolic fuel: a study on the possible influence of teachers' knowledge on the establishment of a misconception among Brazilian high school students.

Advances in Physiology Education, Bethesda, v. 32, n. 3, p. 225-230, 2008.

LUZ, M. R. et al. Glucose as the sole metabolic fuel: the possible influence of formal teaching on the establishment of a misconception about energy-yielding metabolism among students from Rio de Janeiro, Brazil. Biochemistry and Molecular Biology Education, New York, v. 36, n. 6, p. 407-416, 2008.

MARCHIORI, M.; LATORA, V. Harmony in the small-world. Physica A: theoretical and statistical physics, Amsterdam, v. 285, p. 539-546, 2000. Disponible en: <http:/ /arxiv.org/ pdf/cond-mat/0008357.pdf>. Acceso el: 29 enero 2015.

MARÍN MARTÍNEZ, N. Conocimientos que interaccionan con la enseñanza de las ciencias. Enseñanza de las Ciencias, Barcelona, v. 21, n. 1, p. 65-78, 2003.

MÉRIDA, F. Redes cognitivas y sociales: análisis de las estructuras de los textos. Barcelona: Ciudad Educativa, 2004.

OLIVEIRA, G. A. et al. Students' misconception about energy-yielding metabolism: glucose as the sole metabolic fuel. Advances in Physiology Education, Bethesda, v. 27, n. 3, p. 97-101, 2003. Disponible en: <http://advan.physiology.org/content/27/3/97.long>. Acceso el: 29 enero 2015.

QUILLIAN, M. R. Semantic memory. In: MINSKY, M. (Ed.). Semantic information processing. Cambridge: MIT Press, 1968. p. 27-70.

RYLE, G. The concept of mind. New York: Penguin Books, 1949.

SHAPIRO, S. C.; WOODMANSEE, G. H. A net structure based relational question answerer: description and examples. In: INTERNATIONAL JOINT CONFERENCES ON ARTIFICIAL INTELLIGENCE, 1969, Washington. Proceedings... Disponible en: <http:/ /ijcai.org/Past\%20Proceedings/IJCAI-69/PDF/034.pdf>. Acceso el: 29 enero 2015. 
SIGMAN, M.; CECCHI, G. A. Global organization of the wordnet lexicon. Proceedings of the National Academy of Sciences of the United States of America, Washington, v. 99, n. 3, p. 142-247, 2005. Disponible en: <http://www.pnas.org/content/99/3/1742.full. pdf + html>. Acceso el: 29 enero 2015.

SIRVENT, M. T. El proceso de investigación. 2. ed. rev. Buenos Aires: UBA, 2006. (Cuadernos de la Oficina de Publicaciones de la Facultad de Filosofía y Letras). Disponible en: <http://fcp.uncu.edu.ar/claroline/backends/download. php?url=L1VOSURBRF9JL1NpcnZlbnRfRWxfcHJvY2Vzb19kZV9pbnZlc3RpZ2 FjaW9uLnBkZg\%3D\%3D\&cidReset=true\&cidReq=METODOLOGIA $>$. Acceso el: 09 feb. 2015.

SONGER, C.; MINTZES, J. Understanding cellular respiration: an analysis of conceptual changes in college biology. Journal of Research in Science Teaching, Hoboken, v. 31, n. 6, p. 621-637, 1994.

STEYVERS, M.; TENENBAUM, J. B. The large-scale structure of semantic networks: statistical analyses and a model of semantic growth. Cognitive Science, Hoboken, v. 29, n. 1, p. 41-78, 2005.

STRYER, L.; BERG, J. M.; TYMOCZKO, J. L. Bioquímica. 5. ed. Barcelona: Reverté, 2003. TABER, K. S. Classroom-based research and evidence-based practice: a guide for teachers. London: Sage, 2007.

TAMAYO, O. Modelización de procesos de enseñanza en profesores de ciencias de la ciudad de Manizales (Colombia), desde el concepto contenido pedagógico del conocimiento. Enseñanza de las Ciencias, Barcelona, n. 2721, 2009. Número extra.

TIKUNOFF, W. J.; WARD, B. A.; GRIFFIN; G. A. Interactive research and development on teaching study: final report. San Francisco: Far West Laboratory for Educational Research and Development, 1979.

TULVING, E. Episodic and semantic memory. In: TULVING, E.; DONALDSON, W. (Ed.). Organization of memory. New York: Academic Press, 1972. p. 381-402.

VERA-NORIEGA, J. A.; PIMENTEL, C. E.; ALBUQUERQUE, F. J. B. Redes semánticas: aspectos teóricos, técnicos, metodológicos y analíticos. Ra Ximhai, México, v. 1, n. 3, p. 439-451, 2005. Disponible en: <http:/ /www.redalyc.org/articulo.oa?id=46110301>. Acceso el: 03 feb. 2015.

VIVAS, J. R.; COMESAÑA, A.; VIVAS, L.Y. Evaluación de las redes semánticas de conceptos académicos en estudiantes universitarios. PsicoUSF, Itatiba, v. 12, n. 1, p. 111-119, 2007. Disponible en: <http://dx.doi.org/10.1590/S1413-82712007000100013>. Acceso el: 29 enero 2015.

WATTS, D. J.; STROGATZ, S. H. Collective dynamic of "small world" networks. Nature, London, v. 393, p. 440-442, 1998. 